\title{
INVERSION IN INDIRECT OPTIMAL CONTROL
}

\author{
François Chaplais*, Nicolas Petit ${ }^{\dagger}$ \\ * Centre Automatique et Systèmes, École Nationale Supérieure des Mines de Paris, \\ 35, rue Saint-Honoré 77305 Fontainebleau Cedex, France, francois . chaplai s@ensmp. fr \\ $\dagger$ Centre Automatique et Systèmes, École Nationale Supérieure des Mines de Paris, \\ 60, bd. Saint-Michel, 75272 Paris Cedex 06, France, nicolas . petit @ensmp. fr
}

Keywords: Optimal control, indirect methods, adjoint, inversion, nonlinear control theory.

\begin{abstract}
In this paper we explain how to use inversion (as defined in nonlinear control theory) for indirect optimal control. Given the relative degree $r$, it is possible to recover $r$ adjoint states and thus to simplify the problem. Explicit proof is given and relies on the triangular structure of the underlying normal form. An example from the literature is treated in the last section.
\end{abstract}

\section{Introduction}

Geometric tools have long been used in control theory for feedback linearization $[5,8]$. The induced change of variables lead to straightforward resolution of inverse problem, i.e. computation of required inputs for a prescribed behavior of outputs. Optimization of the obtainable trajectories is an important topic, especially for applications, but generally requires numerical solvers. Inversion has lately been used in numerical optimal control and the numerical importance of the relative degree of the output chosen when casting the optimal control problem into a numerical collocation scheme was emphasized in [9]. It yields substantial reduction in required CPU time [7]. The bestcase scenario is full feedback linearization (flatness) as considered in [3].

Given the system dynamics and an optimal cost, it was shown how to take advantage of the geometric structure of the dynamics to reduce the dimensionality of a numerical collocation scheme. In general collocation methods, coefficients are used to approximate with basis functions both states and inputs [4]. While it was known since [11] that it is numerically efficient to eliminate the control, it was emphasized in [9] that it is possible to reduce the problem further. Choosing outputs with maximum relative degrees is the key to efficient variable elimination that makes the required number of coefficients smaller. When combined to a nonlinear programming solver, this can induce drastic speed-ups in numerical solving [6].

In this paper we focus on indirect methods (i.e. adjoint methods) as opposed to the previously discussed direct collocation schemes. We show that in this framework it is also possible to take advantage of the geometric structure of the dynamics. More precisely we show that given a SISO system with a $n$ dimensional state, the relative degree of the system also plays a role in its dual dynamics. We derive an equivalent rewriting of the dynamics of the two-point boundary value problem where we eliminate as many variables as we can. In the case of full feedback linearisability (flatness) the induced equation is a $2 n$-degree differential equation in the linearizing output.

The article organizes as follows. In section 1 we detail the problem we address (SISO system with generic terminal and integral cost). In section 2 we explain how to recover the geometric structure of the adjoint equation. Further we detail how to take boundary conditions into account for two special cases: terminal cost with or without terminal constraints. In section 3 we treat a numerical example from the literature.

\section{Problem settings}

Consider an optimal control problem with dynamics

$$
\frac{d x}{d t}=f(x)+g(x) u, u \in \mathbb{R}, x \in \mathbb{R}^{n}
$$

where all vector fields and functions are real-analytic.

It is desired to find a trajectory of (1), i.e. $\left[0, t_{f}\right] \ni t \mapsto$ $(x, u)(t) \in \mathbb{R}^{n+1}$, that minimizes the performance index

$$
\min \phi\left(x\left(t_{f}\right)\right)+\int_{0}^{t_{f}} L(x(t), u(t)) d t
$$

where $L$ is a smooth nonlinear function

A (SISO) single input single output system

$$
\begin{aligned}
\frac{d x}{d t} & =f(x)+g(x) u \\
y & =h(x)
\end{aligned}
$$

is said to have relative degree (see for instance [5]) $r$ at point $x_{0}$ if $L_{g} L_{f}^{k} h(x)=0$, in a neighborhood of $x_{0}$ and for all $k<r-1$, and $L_{g} L_{f}^{r-1} h\left(x_{0}\right) \neq 0$ where $L_{f} h(x)=\sum_{i=1}^{n} \frac{\partial h}{\partial x_{i}} f_{i}(x)$ is the derivative of $h$ along $f$. Roughly speaking, $r$ is the number of times one has to differentiate $y$ before $u$ appears.

An important result (see [5]) is as follows. Suppose the system (3) has relative degree $r$ at $x^{0}$. Then $r \leq n$. Set

$$
\begin{aligned}
\phi_{1}(x) & =h(x) \\
\phi_{2}(x) & =L_{f} h(x) \\
& \vdots \\
\phi_{r}(x) & =L_{f}^{r-1} h(x)
\end{aligned}
$$


If $r$ is strictly less than $n$, it is always possible to find $n-r$ more functions $\phi_{r+1}(x), \ldots, \phi_{n}(x)$ such that the mapping

$$
\phi(x)=\left(\begin{array}{c}
\phi_{1}(x) \\
\vdots \\
\phi_{n}(x)
\end{array}\right)
$$

has a Jacobian matrix which is nonsingular at $x^{0}$ and therefore qualifies as a local coordinates transformation in a neighborhood of $x^{0}$. The value at $x^{0}$ of these additional functions can be fixed arbitrarily. Moreover, it is always possible to choose $\phi_{r+1}(x), \ldots, \phi_{n}(x)$ in such a way that $L_{g} \phi_{i}(x)=0$, for all $r+1 \leq i \leq n$ and all $x$ around $x^{0}$.

The implication of this result is that there exists a change of coordinates

$$
x \mapsto\left(z_{1}, z_{2}, \ldots, z_{r}, \eta\right) \equiv(\zeta, \eta) \in \mathbb{R}^{n}
$$

such that the systems equations may be written under the following normal form

$$
\begin{aligned}
\frac{d z_{i}}{d t} & =z_{i+1} \text { for } i=1 \ldots r-1 \\
\frac{d z_{r}}{d t} & =b(\zeta, \eta)+a(\zeta, \eta) u \\
\frac{d \eta}{d t} & =q(\zeta, \eta)
\end{aligned}
$$

where $a(\zeta, \eta)$ is nonzero for all $(\zeta, \eta)$ in a neighborhood of $(\zeta, \eta)=\phi\left(x^{0}\right)$.

The inverse change of coordinates gives

$$
\begin{aligned}
x(t) & =A(\zeta(t), \eta(t)) \\
u(t) & =B\left(\zeta(t), \frac{d z_{r}}{d t}, \eta(t)\right)
\end{aligned}
$$

In the coordinates $(\zeta, \eta),(2)$ is rewritten as

$$
\min \phi\left(A\left(\zeta\left(t_{f}\right), \eta\left(t_{f}\right)\right)\right)+\int_{0}^{t_{f}} L(A(\zeta(t), \eta(t)), u(t)) d t
$$

with dynamics $(4,5,6)$ (when one does not substitute $u$ using (8)).

\section{Hamiltonian computations}

\subsection{The Hamiltonian}

Because of the triangular structure of the previous normal form, the Hamiltonian of cost $(9)$ with dynamics $(4,5,6)$ is

$$
\begin{aligned}
H(\zeta, \eta, u, \lambda, \mu)= & L(A(\zeta, \eta), u)+\sum_{i=1}^{i=r-1} \lambda_{i} z_{i+1} \\
& +\lambda_{r}[b(\zeta, \eta)+a(\zeta, \eta) u]+\mu^{T} q(\zeta, \eta)
\end{aligned}
$$

\subsection{Adjoint equations}

The necessary conditions of Pontryaguin on the Hamiltonian imply

$$
\begin{aligned}
& \frac{d \lambda_{1}}{d t}=-\frac{\partial L}{\partial A} \frac{\partial A}{\partial z_{1}}-\lambda_{r}\left[\frac{\partial b}{\partial z_{1}}+\frac{\partial a}{\partial z_{1}} u\right]-\mu^{T} \frac{\partial q}{\partial z_{1}} \\
& \frac{d \lambda_{i}}{d t}=-\frac{\partial L}{\partial A} \frac{\partial A}{\partial z_{i}}-\lambda_{i-1}-\lambda_{r}\left[\frac{\partial b}{\partial z_{i}}+\frac{\partial a}{\partial z_{i}} u\right]-\mu^{T} \frac{\partial q}{\partial z_{i}} \\
& \quad \text { for } i=2 \ldots r \\
& \frac{d \mu_{i}}{d t}=-\frac{\partial L}{\partial A} \frac{\partial A}{\partial \eta_{i}}-\lambda_{r}\left[\frac{\partial b}{\partial \eta_{i}}+\frac{\partial a}{\partial \eta_{i}} u\right]-\mu^{T} \frac{\partial q}{\partial \eta_{i}}
\end{aligned}
$$

on the optimal trajectory.

\subsection{Optimality conditions}

Since there is no constraint on the control $u, \frac{\partial H}{\partial u}$ is necessary zero along the optimal trajectory. Observe that this partial derivative does not involve the adjoint states $\left(\lambda_{1}, \ldots, \lambda_{r-1}, \mu\right)$, i. e. , there is a function $G_{0}\left(z_{1}, \ldots, z_{r}, u, \eta\right)$ such that

$$
\frac{\partial H}{\partial u}=G_{0}\left(z_{1}, \ldots, z_{r}, u, \eta\right)+\lambda_{r} a(\zeta, \eta)=0
$$

along the optimal trajectory. Inserting (8) into (13) shows that there exists $F_{0}$ such that

$$
\frac{\partial H}{\partial u}=F_{0}\left(z_{1}, \ldots, z_{r}, \frac{d z_{r}}{d t}, \eta, \lambda_{r}\right)=0
$$

along the optimal trajectory.

Its derivatives are thus also zero along the trajectory. Their structure is given by the following lemma

Lemma 1. For $i>r$, let $z_{i}(t)=\frac{d^{r-i} z_{r}}{d t^{r-i}}=\frac{d^{i-1} z_{1}}{d t^{i-1}}$. For $i=$ $1 \ldots r-1$, the $i^{\text {th }}$ time derivative of $\frac{\partial H}{\partial u}$ along the optimal trajectory has the structure

$$
\frac{d^{i}}{d t^{i}}\left(\frac{\partial H}{\partial u}\right)=F_{i}\left(z_{1}, \ldots, z_{r+i+1}, \eta, \lambda_{r-i}, \ldots, \lambda_{r}, \mu\right)
$$

where $F_{i}$ is affine with respect to $\left(\lambda_{r-i}, \ldots, \lambda_{r}, \mu\right)$ and satisfies

$$
\frac{\partial F_{i}}{\partial \lambda_{r-i}}=(-1)^{i} a\left(z_{1}, \ldots, z_{r}, \eta\right)
$$

Proof. Equation (13) gives the desired result for $i=0$. The proof proceeds then by induction. Let us assume that (15) holds for $i<r-1$. Taking one more derivative of (15) and using (8) yields equation (17). This last function is of the form (15).

If $F_{i}$ is affine with respect to $\left(\lambda_{r-i}, \ldots, \lambda_{r}, \mu\right)$, then the previous computations show that $F_{i+1}$ is affine with respect to $\left(\lambda_{r-i-1}, \ldots, \lambda_{r}, \mu\right)(H$ is also affine with respect to the adjoint states). Observe that $\frac{d \lambda_{1}}{d t}$ does not appear in the computations since $r-i>1$. Also, $\lambda_{r-i-1}$ only appears once (for $j=r-i$ ) and the derivative of the right handside expression with respect to it is $-\frac{\partial F_{i}}{\partial \lambda_{r-i}}$, which proves (16) for $i+1$. 


$$
\begin{aligned}
\frac{d^{i+1}}{d t^{i+1}}\left(\frac{\partial H}{\partial u}\right)= & \sum_{j=1}^{r+i+1} \frac{\partial F_{i}}{\partial z_{j}} z_{j+1}+\frac{\partial F_{i}}{\partial \eta} q+\sum_{j=r-i}^{r} \frac{\partial F_{i}}{\partial \lambda_{j}}\left[-\frac{\partial L}{\partial A} \frac{\partial A}{\partial z_{j}}-\lambda_{j-1}-\lambda_{r}\left[\frac{\partial b}{\partial z_{j}}+\frac{\partial a}{\partial z_{j}} u\right]-\mu^{T} \frac{\partial q}{\partial z_{j}}\right] \\
& -\frac{\partial F_{i}}{\partial \mu}\left(z_{1}, \ldots, z_{r+i+1}, \eta\right)\left(\frac{\partial H}{\partial \eta}\right)^{T}\left(\zeta, \eta, \lambda_{r}, \mu\right) \\
= & \sum_{j=1}^{r+i+1} \frac{\partial F_{i}}{\partial z_{j}} z_{j+1}+\frac{\partial F_{i}}{\partial \eta} q \\
& +\sum_{j=r-i}^{j=r} \frac{\partial F_{i}}{\partial \lambda_{j}}\left[-\frac{\partial L}{\partial A} \frac{\partial A}{\partial z_{j}}-\lambda_{j-1}-\lambda_{r}\left[\frac{\partial b}{\partial z_{j}}+\frac{\partial a}{\partial z_{j}} B\left(z_{1}, \ldots, z_{r+1}, \eta\right)\right]-\mu^{T} \frac{\partial q}{\partial z_{i}}\right]-\frac{\partial F_{i}}{\partial \mu}\left(\frac{\partial H}{\partial \eta}\right)^{T} \\
\equiv & F_{i+1}\left(z_{1}, \ldots, z_{r+i+2}, \eta, \lambda_{r-i-1}, \ldots, \lambda_{r}, \mu\right)
\end{aligned}
$$

The adjoint states $\left(\lambda_{1}, \ldots, \lambda_{r}\right)$ corresponding to the invertible part of the normal form (or upper part) can be eliminated from (15) along the optimal trajectory.

Lemma 2. For $i=0 \ldots r-1$, there exists a function $W_{i}$ such that

$$
\lambda_{r-i}=W_{i}\left(z_{1}, \ldots, z_{r+i+1}, \eta, \mu\right)
$$

along the optimal trajectory at times where $a\left(z_{1}, \ldots, z_{r}\right)$ is not zero. For concision we shall note $\lambda=W\left(z_{1}, \ldots, z_{2 r}, \eta, \mu\right)$.

Proof. Gathering the conditions $H_{u}=0$ and $\frac{d^{i}}{d t^{i}}\left(\frac{\partial H}{\partial u}\right)=0$ for $i=1, \ldots, r-1$, one get the following system of $r$ equations

$$
\begin{array}{r}
F_{0}\left(z_{1}, \ldots, \frac{d^{r+1} z_{1}}{d t^{r+1}}, \eta, \lambda_{r}\right)=0 \\
F_{1}\left(z_{1}, \ldots, \frac{d^{r+2} z}{d t^{r+2}}, \eta, \lambda_{r-1}, \lambda_{r}, \mu\right)=0 \\
\vdots \\
F_{r-1}\left(z_{1}, \ldots, \frac{d^{2 r} z}{d t^{2 r}}, \eta, \lambda_{1}, \ldots, \lambda_{r}, \mu\right)=0
\end{array}
$$

The system $\left(F_{i}=0\right)$ is linear and triangular with respect to the $\lambda_{i}$. Up to the sign, the diagonal term is $\frac{\partial F_{0}}{\partial \lambda_{r}}=a\left(z_{1}, \ldots, z_{r}\right)$. Hence the system $\left(F_{i}=0\right)$ has a unique solution with respect to the $\lambda_{i}$. For $i=0$, equation (18) is easily drawn from (13). Consider now $F_{i}=0$ for $i>0$. From (15) we see that $\lambda_{r-i}$ is a function of $\left(z_{1}, \ldots, z_{r+i+1}, \lambda_{r-i+1}, \ldots, \lambda_{r}\right)$; for $j<i$, we assume recursively that $\lambda_{r-j}$ is a function $W_{j}$ of $\left(z_{1}, \ldots, z_{r+j+1}\right)$, with $r+j+1<r+i+1$. Hence $\lambda_{r-i}$ is a function $W_{i}$ of $\left(z_{1}, \ldots, z_{r+j+1}\right)$.

For $i=r-1$, we have

$$
\lambda_{1}(t)=W_{r-1}\left(z_{1}(t), \ldots, z_{2 r}(t), \eta, \mu\right)
$$

Differentiating this equation and using the adjoint equation on $\lambda_{1}$ leads to
Proposition (Main result). The optimal output $z_{1}$ satisfies the following differential equation of order $(2 r)$ :

$$
\begin{aligned}
\sum_{i=1}^{i=2 r} & \frac{\partial W_{r-1}}{\partial z_{i}} z_{i+1}+\frac{\partial W_{r-1}}{\partial \eta} q-\frac{\partial W_{r-1}}{\partial \mu}\left(\frac{\partial H}{\partial \eta}\right)^{T}= \\
& -\mu^{T} \frac{\partial q}{\partial z_{1}}-\frac{\partial L}{\partial A} \frac{\partial A}{\partial z_{1}} \\
& -W_{0}\left(z_{1}, \ldots, z_{r+1}, \eta\right)\left[\frac{\partial b}{\partial z_{1}}+\frac{\partial a}{\partial z_{1}} B\left(z_{1}, \ldots, z_{r+1}, \eta\right)\right]
\end{aligned}
$$

with $z_{i}(t)=\frac{d^{i-1} z_{1}}{d t^{i-1}}$.

Conversely, assume that $z_{1}$ satisfies (20); let $\eta$ a solution of (6) and $\mu$ a solution of

$$
\begin{aligned}
\frac{d \mu_{i}}{d t}= & -\frac{\partial H}{\partial \eta_{i}}-\frac{\partial L}{\partial A} \frac{\partial A}{\partial \eta_{i}} \\
& -W_{0}\left(z_{1}, \ldots, z_{r+1}, \eta\right)\left[\frac{\partial b}{\partial \eta_{i}}+\frac{\partial a}{\partial \eta_{i}} u\right]-\mu^{T} \frac{\partial q}{\partial \eta_{i}}
\end{aligned}
$$

with

$$
u=\frac{z_{r+1}-b\left(z_{1}, \ldots, z_{r}\right)}{a\left(z_{1}, \ldots, z_{r}\right)}
$$

Define $\Lambda=W\left(z_{1}, \ldots z_{2 r}, \eta, \mu\right)$ and $\zeta=\left(z_{1}, \ldots z_{r}\right)$. If $\lambda=$ $\left(\lambda_{1}, \ldots \lambda_{r}\right)$ satisfies $(10,11)$ and $\partial H / \partial u(\zeta, \eta, u, \lambda, \mu)=0$, then $\zeta$ satisfies (4,5), $\lambda=\Lambda$ and $\mu$ satisfies (12).

Proof. The left handside is the time derivative of (19). The right handside is the insertion of (8) and of (18) for $i=0$ into (10).

The converse part essentially shows that $\lambda$ can be drawn from $z_{1}$ and its derivatives and, of course $\eta$ and $\mu$. It is closely related to the direct part. From the definition (22) of $u$, it follows that $\zeta$ satisfies (4,5). Since $\partial H / \partial u=0$, then $\lambda_{r}$ satisfies (13), and hence, $\lambda_{r}=W_{0}\left(z_{1}, \ldots, z_{r+1}, \eta\right)=\Lambda_{r}$. Together with (21), it follows that $\mu$ satisfies (12). Since $\partial H / \partial u=0$, it follows from lemmas 1 and 2 that $\lambda=W\left(z_{1}, \ldots, z_{2 r}, \eta, \mu\right)=\Lambda$. 
In other words the $1+2(n-r)$ unknowns to be found are $z_{1}, \eta$, and $\mu$. Then the $\Lambda$ as defined above are appropriate candidates for the adjoint states corresponding to $\left(z_{1}, \ldots z_{r}\right)$ (the only adjoint states that are missing).

\subsection{Endpoint conditions}

With the proposed terminal cost $\phi\left(x\left(t_{f}\right)\right)=$ $\phi\left(A\left(z_{1}, \ldots, z_{r}, \eta\right)\right)$, the terminal conditions on the adjoint states are then

$$
\lambda_{i}\left(t_{f}\right)=\frac{\partial}{\partial z_{i}} \phi\left(A\left(z_{1}\left(t_{f}\right), \ldots, z_{r}\left(t_{f}\right), \eta\left(t_{f}\right)\right)\right), \text { for } i=1, \ldots, r
$$

That can be rewritten in terms of conditions upon the derivatives of the flat output

$$
\begin{aligned}
& W_{i}\left(z_{1}\left(t_{f}\right), \ldots, z_{r+i+1}\left(t_{f}\right), \eta\left(t_{f}\right), \mu\left(t_{f}\right)\right)= \\
& \frac{\partial}{\partial z_{r-i}} \phi\left(A\left(z_{1}\left(t_{f}\right), \ldots, z_{r}\left(t_{f}\right), \eta\left(t_{f}\right)\right)\right),
\end{aligned}
$$

for $i=1, \ldots, r$

\subsection{Endpoint equality constraints}

Following Bryson and Ho's [2] presentation of optimal control problems we explain how to treat a special case of practical interest. Our proposed approach applies in this situation where extra adjoint states appear.

Let us consider the original problem (1), (2) with the additional constraint

$$
\psi\left(x\left(t_{f}\right)\right)=0 .
$$

Where $\psi$ is a smooth function from $\mathbb{R}^{n} \rightarrow \mathbb{R}^{q}, 0<q<n$. Introducing $q$ extra multipliers $\pi \in \mathbb{R}^{q}$ we get the terminal conditions

$$
\begin{aligned}
\lambda_{i}\left(t_{f}\right)= & \frac{\partial}{\partial z_{i}} \phi\left(A\left(z_{1}\left(t_{f}\right), \ldots, z_{r}\left(t_{f}\right), \eta\left(t_{f}\right)\right)\right) \\
& +\pi^{T} \frac{\partial}{\partial z_{i}} \psi\left(A\left(z_{1}\left(t_{f}\right), \ldots, z_{r}\left(t_{f}\right), \eta\left(t_{f}\right)\right)\right)
\end{aligned}
$$

for $i=1, \ldots, r$ along with the $q$ terminal constraints (23). Under the assumption that one can extract a non singular $q \times q$ matrix out of the Jacobian of $\psi$ (which is a classical assumption to make (23) solvable), it is possible to isolate an invertible set of $q$ equations in the $q$ unknows $\pi$ from the set of equations (24). This means that these multipliers also write in terms of $z_{1}$ and its successive derivatives, $\eta$ and $\mu$. Let $\left(i_{1}, \ldots, i_{q}\right)$ be a set of indexes such that the $q \times q$ matrix extracted from the Jacobian of $\psi$ is invertible, let $M_{q}$ denote this matrix extracted from

$$
\left(\frac{\partial}{\partial z} \psi\left(A\left(z_{1}\left(t_{f}\right), \ldots, z_{r}\left(t_{f}\right), \eta\left(t_{f}\right)\right)\right)\right)
$$

This matrix depends on $z_{1}\left(t_{f}\right), \ldots, z_{r}\left(t_{f}\right), \eta\left(t_{f}\right)$. Let $\bar{W}$ denote the vector $\left(W_{i_{k}}\right)$, for $k=1, \ldots, q$ and $\bar{z}$ the vector $\left(z_{i_{k}}\right)$, for $k=1, \ldots, q$. Then one can solve

$$
\begin{aligned}
\pi=M_{q}^{-1} & \left(\bar{W}\left(z_{1}\left(t_{f}\right), \ldots, z_{r}\left(t_{f}\right), \eta\left(t_{f}\right), \mu\left(t_{f}\right)\right)^{T}\right. \\
& \left.-\left(\frac{\partial}{\partial \bar{z}}\right) \phi\left(A\left(z_{1}\left(t_{f}\right), \ldots, z_{r}\left(t_{f}\right), \eta\left(t_{f}\right)\right)\right)\right)
\end{aligned}
$$

\section{Example}

To illustrate the previous result we derive here the optimality conditions for the forced van der Pol Problem.

The dynamics of this system discussed in [7] and [10] is given by

$$
\begin{aligned}
& \dot{x}_{1}=x_{2} \\
& \dot{x}_{2}=-x_{1}+\left(1-x_{1}^{2}\right) x_{2}+u
\end{aligned}
$$

It was used in the last two cited references as a benchmark. The optimal control problem is to minimize the quadratic cost function

$$
J=\int_{0}^{5}\left(x_{1}^{2}(s)+x_{2}^{2}(s)+u^{2}(s)\right) d s
$$

subject to the dynamics given above and the endpoint constraints

$$
x_{1}(0)=1, x_{2}(0)=0, x_{2}(5)-x_{1}(5)=1
$$

The dynamics is flat and $z_{1}=x_{1}$ is a flat output (ideal case). Following the procedure presented in the previous section we get the Hamiltonian

$H=\frac{1}{2}\left(x_{1}^{2}+x_{2}^{2}+u^{2}\right)+\lambda_{1} x_{2}+\lambda_{2}\left(-x_{1}+\left(1-x_{1}^{2}\right) x_{2}+u\right)$

The adjoint equations are

$$
\begin{aligned}
& \dot{\lambda}_{1}=-H_{x_{1}}=-\left(x_{1}-2 \lambda_{2} x_{1} x_{2}\right) \\
& \dot{\lambda}_{2}=-H_{x_{2}}=-\left(x_{2}+\lambda_{1}+\lambda_{2}\left(1-x_{1}^{2}\right)\right)
\end{aligned}
$$

Solving the two equations $H_{u}=0$ and $\frac{d H_{u}}{d t}=0$ for the adjoint states we get

$$
\begin{aligned}
\lambda_{1}= & x_{1}^{(3)}+\dot{x}_{1}+2 x_{1} \dot{x}_{1}^{2}-\left(1-x_{1}^{2}\right) \ddot{x}_{1} \\
& -\dot{x}_{1}-\left(-x_{1}^{(2)}-x_{1}+\left(1-x_{1}^{2}\right) \dot{x}_{1}\right)\left(1-x_{1}^{2}\right) \\
\lambda_{2}= & -x_{1}^{(2)}-x_{1}+\left(1-x_{1}^{2}\right) \dot{x}_{1}
\end{aligned}
$$

Now one just has to substitute the expression for $\lambda_{1}$ and $\lambda_{2}$ into the differential equation (25) to get the fourth order differential equation to be satisfied by the flat output on the optimal trajectory

$$
\begin{aligned}
& x_{1}^{(4)}+2 x_{1}^{3}+6 x_{1} \dot{x}_{1} \ddot{x}_{1}+ \\
& \left(2 x_{1} \dot{x}_{1}-\left(1-x_{1}^{2}\right) \ddot{x}_{1}\right)\left(1-x_{1}^{2}\right)+2 x_{1}+\ddot{x}_{1}=0
\end{aligned}
$$


Finally one gets the boundary conditions

$$
\begin{aligned}
x_{1}(0) & =1 \\
\dot{x}_{1}(0) & =0 \\
\dot{x}_{1}(5)-x_{1}(5) & =0 \\
x_{1}^{(3)}(5)+\dot{x}_{1}(5)+2 & x_{1}(5) \dot{x}_{1}^{2}(5)-\left(1-x_{1}^{2}(5)\right) \ddot{x}_{1}(5)-\dot{x}_{1}(5) \\
-\left(-x_{1}^{(2)}(5)-x_{1}(5)\right. & \left.+\left(1-x_{1}^{2}(5)\right) \dot{x}_{1}(5)\right)\left(1-x_{1}^{2}(5)\right) \\
& =x_{1}^{(2)}(5)+x_{1}(5)-\left(1-x_{1}^{2}(5)\right) \dot{x}_{1}(5)
\end{aligned}
$$

the last relation being just the endpoint condition $\lambda_{1}(5)+$ $\lambda_{2}(5)=0$ expressed in terms of the flat output.

Solving this two point boundary value problem can be done with any appropriate numerical solver, e.g. Matlab bvp4c. Results give solutions comparable to the one obtained in [7] and [10], the obtained cost is 1.68588 .

The adjoint states $\lambda_{1}$ and $\lambda_{2}$ also writes in terms of the flat output along the optimal trajectory as given by (26) and (27).

\section{Conclusion}

In summary, what we have shown is the following: if the system has relative degree $r$, then $r$ of the adjoint states can be explicitly recovered. To do so one just has to rewrite the problem in its normal form and then write that $H_{u}$ and its first $r-1$ time derivatives are zero along the optimal trajectories.

As stated in the main result of the paper, finding an optimal trajectory is equivalent to finding time functions $z_{1}(t) \in \mathbb{R}$, $\eta(t) \in \mathbb{R}^{n-r}$ and $\mu(t) \in \mathbb{R}^{n-r}$ that satisfy (20), (6) and (21). We have fewer unknowns than the classical $n$ states and $n$ adjoint states. We are now left with $1+2(n-r)$ unknowns. The ideal case is (as for collocation methods) the fully invertible case (flat case) where $r=n$, as treated in the example in the previous section. In any case we believe this structure could be exploited in implementation of shooting techniques (see [1] for an overview of shooting methods) as it involves fewer unknowns. Numerical relevance of this approach is currently under investigation.

\section{References}

[1] F. Bonnans. The shooting algorithm for optimal control problems: A review of some theoretical and numerical aspects. Lecture notes, http: //www-rocq.inria.fr/sydoco/ cours/tutorial.html, INRIA, 2002.

[2] A. E. Bryson. Dynamic optimization. Addison Wesley, 1999.

[3] N. Faiz, S. Agrawal, and R. M. Murray. Differentially flat systems with inequality constraints: An approach to realtime feasible trajectory generation. J. Guidance, Control, and Dynamics, 24(2):219-227, 2001.
[4] C. Hargraves and S. Paris. Direct trajectory optimization using nonlinear programming and collocation. AIAA J. Guidance and Control, 10:338-342, 1987.

[5] A. Isidori. Nonlinear Control Systems. Springer, New York, 2nd edition, 1989.

[6] M. B. Milam, R. Franz, and R. M. Murray. Real-time constrained trajectory generation applied to a flight control experiment. In Proc. of the IFAC World Congress, 2002.

[7] M. B. Milam, K. Mushambi, and R. M. Murray. A new computational approach to real-time trajectory generation for constrained mechanical systems. In IEEE Conference on Decision and Control, 2000.

[8] H. Nijmeijer and A.J. van der Schaft. Nonlinear Dynamical Control Systems. Springer-Verlag, 1990.

[9] N. Petit, M. B. Milam, and R. M. Murray. Inversion based constrained trajectory optimization. In 5th IFAC symposium on nonlinear control systems, 2001.

[10] M. I. Ross and F. Fahroo. Pseudospectral methods for optimal motion planning of differentially flat systems. In Proc. of the 41th IEEE Conf. on Decision and Control, 2002.

[11] H. Seywald. Trajectory optimization based on differential inclusion. J. Guidance, Control and Dynamics, 17(3):480-487, 1994. 